\title{
Role of Defects in Ion Transport in Block
}

\section{Copolymer Electrolytes: Supporting Information}

Yu Kambe,${ }^{a, b}$ Christopher G. Arges, ${ }^{c}$ David A. Czaplewski, ${ }^{d}$ Moshe Dolejsi, ${ }^{a, b}$ Satya Krishnan, ${ }^{a}$ Mark P. Stoykovich, ${ }^{a}$ Juan J. de Pablo, ${ }^{a, b}$ and Paul F. Nealey ${ }^{a, b, *}$

aPritzker School of Molecular Engineering, University of Chicago, 5640 S. Ellis Ave., Chicago, IL 60637

${ }^{\mathrm{b}}$ Center for Molecular Engineering, Materials Science Division, Argonne National Laboratory, 9700 S. Cass Ave., Lemont, IL 60439

${ }^{\mathrm{c}}$ Cain Department of Chemical Engineering, Louisiana State University, Baton Rouge, LA 70803

${ }^{\mathrm{d}}$ Center for Nanoscale Materials, Argonne National Laboratory, 9700 S. Cass Ave., Lemont, IL 60439

*Corresponding author email: nealey@uchicago.edu 


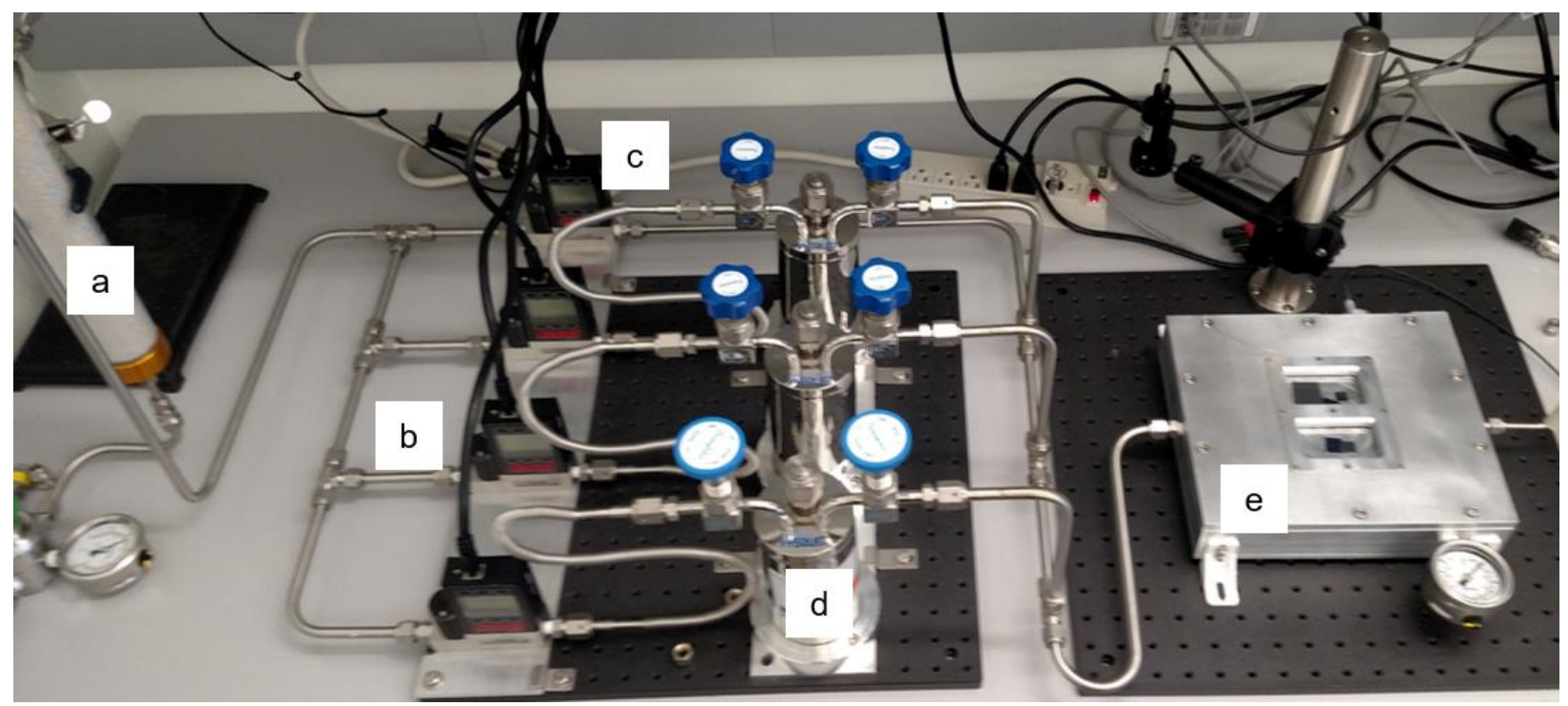

Figure S1. Design of solvent annealing chamber and features. $a, \mathrm{~N}_{2}$ inlet $\mathrm{b}$, mass flow controllers c, control $\mathrm{N}_{2}$ line to chamber $\mathrm{d}$, solvent bubblers e, annealing chamber with quartz crystal and reflectance-based thickness metrology capabilities. 


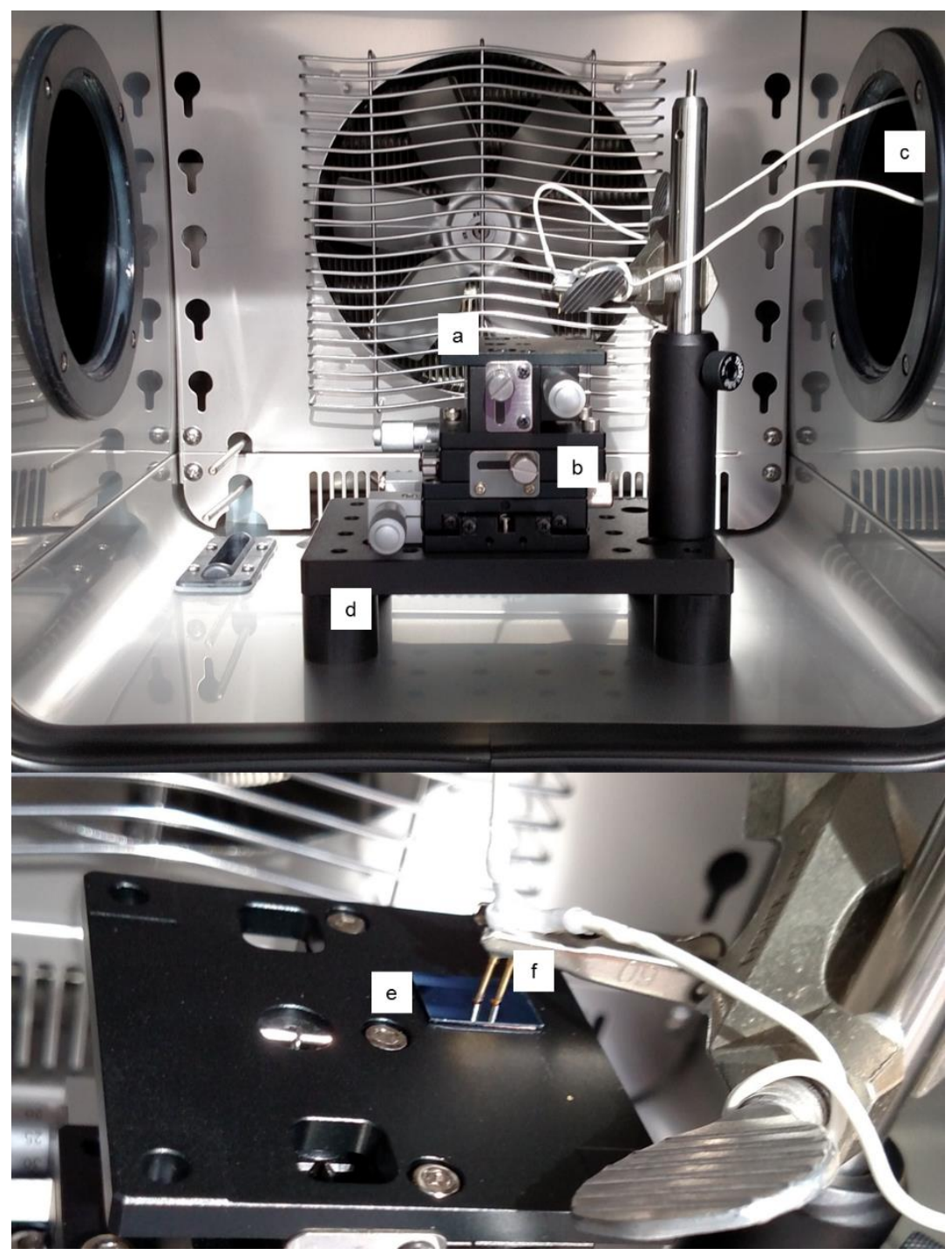

Figure S2. Temperature and humidity controlled electrochemical analysis chamber. a, sample stage b, 3-axis stage c, electrical outlet from the humidity chamber d, vibration isolating support e, sample f, 2-point spring probe contact. 


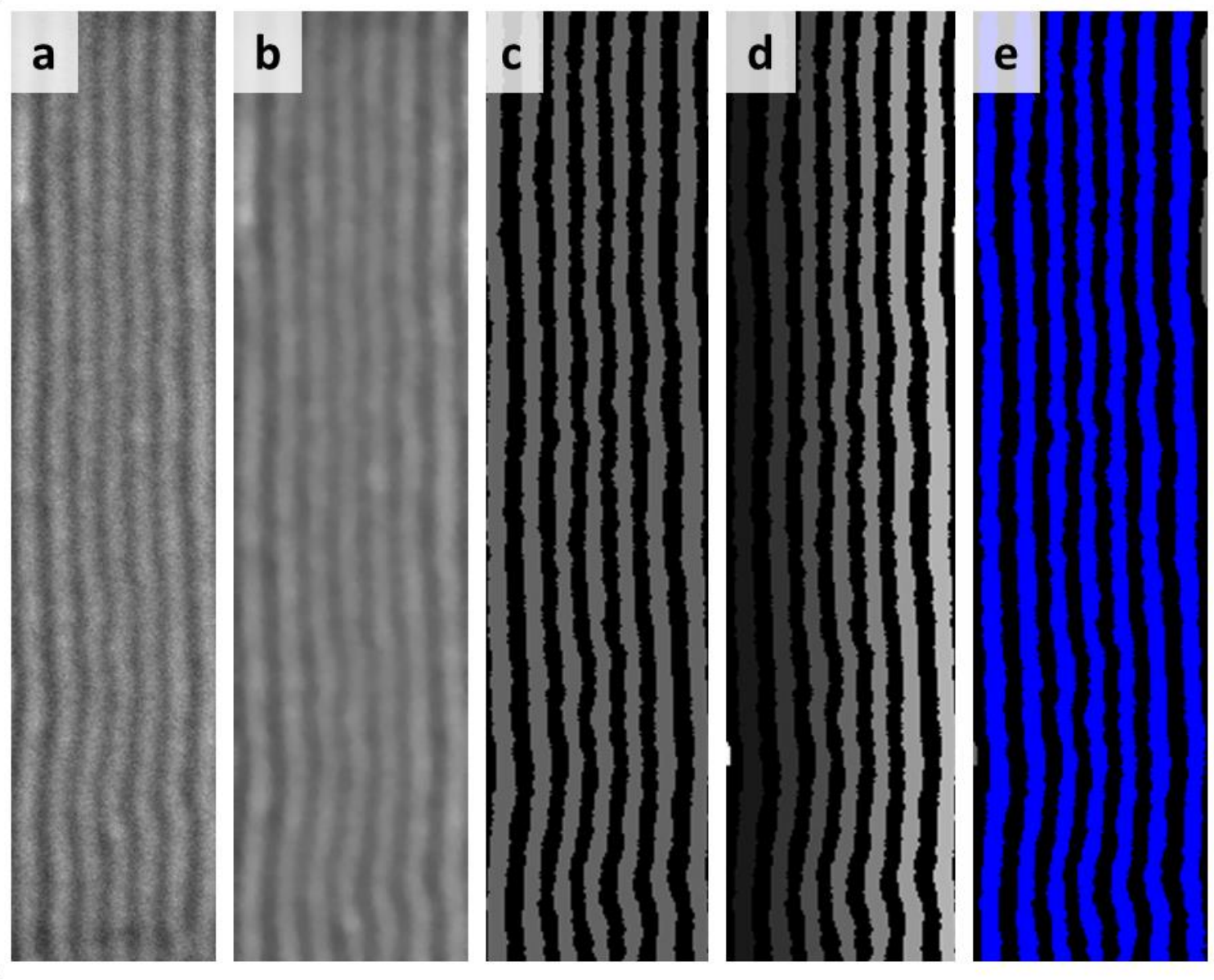

Figure S3. Quantification of structure parameters. a, original SEM image b, denoised image c, threshold image d, labeled image e, interconnected domains which span from one electrode to the other 

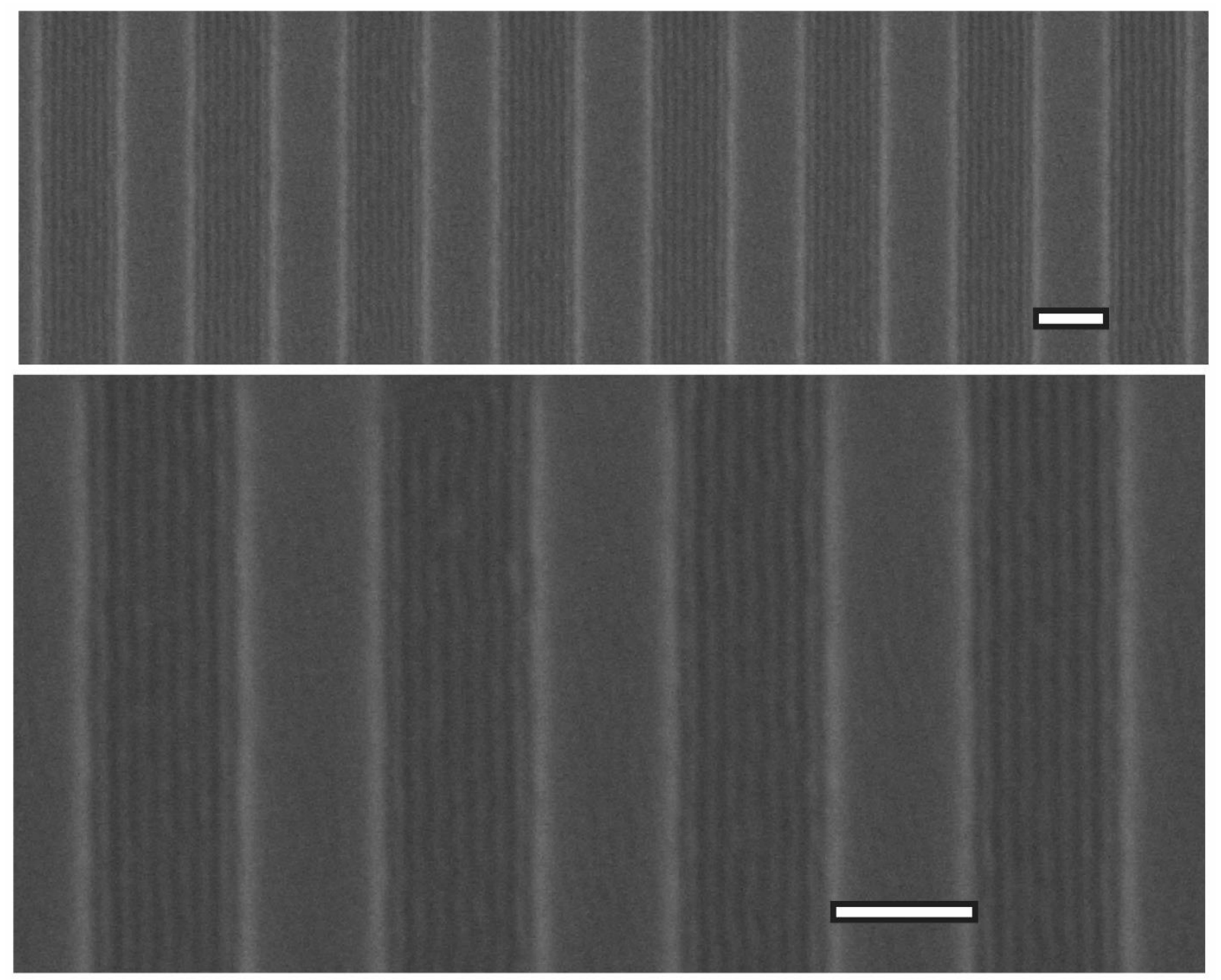

Figure S4. SEM image of the DSA. Scale is $200 \mathrm{~nm}$. 

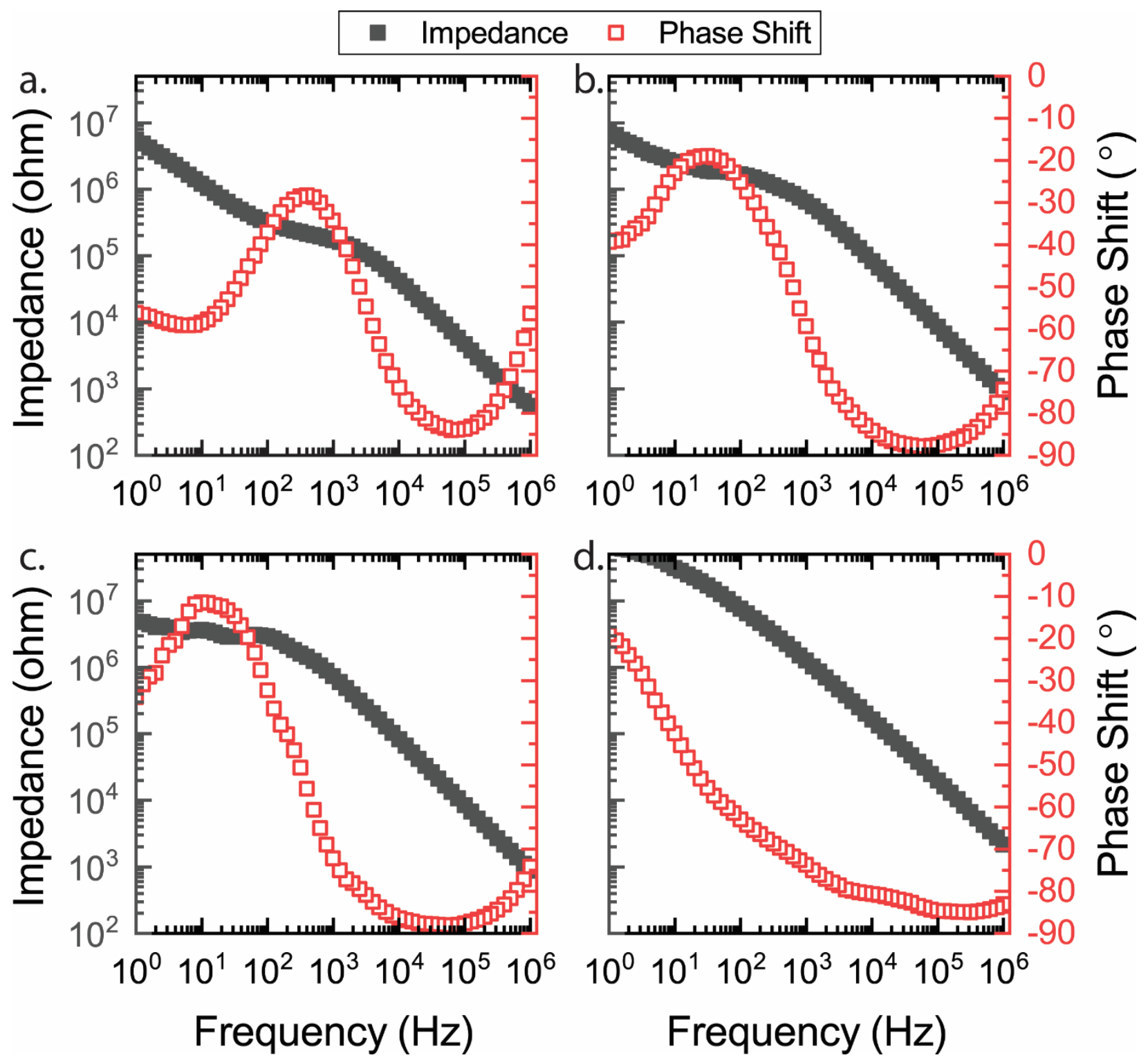

Figure S5. Bode plots for a.) connected b.) partially connected c.) fingerprint and d.) unconnected. 
Table S1. Values extracted from the Bode and Nyquist plots.

\begin{tabular}{|c|c|c|c|c|c|}
\hline & Units & Connected & $\begin{array}{c}\text { Partially } \\
\text { Connected }\end{array}$ & Fingerprint & Unconfined \\
\hline$R_{\mathrm{e}}$ & ohm & 191.4 & 200 & $5.78 \mathrm{E}-01$ & $4.11 \mathrm{E}-03$ \\
\hline$Q_{\mathrm{DL}}$ & $\begin{array}{c}\mathrm{S} \mathrm{s}^{\mathrm{a}} \\
{\left[\mathrm{Siemens} \mathrm{sec}^{\mathrm{a}}\right]}\end{array}$ & $6.36 \mathrm{E}-09$ & $2.75 \mathrm{E}-08$ & 4.87E-08 & $4.16 \mathrm{E}-10$ \\
\hline$a_{\mathrm{DL}}$ & - & $6.94 \mathrm{E}-01$ & $9.66 \mathrm{E}-01$ & $6.85 \mathrm{E}-01$ & $9.00 \mathrm{E}-01$ \\
\hline$Q_{\text {FILM }}$ & $\begin{array}{c}\mathrm{S} \mathrm{s}^{\mathrm{a}} \\
{[\text { Siemens sec }}\end{array}$ & $3.57 \mathrm{E}-11$ & $2.30 \mathrm{E}-10$ & $2.34 \mathrm{E}-10$ & $1.27 \mathrm{E}-09$ \\
\hline$a_{\mathrm{FILM}}$ & - & 1 & $9.86 \mathrm{E}-01$ & 1 & 8.97E-01 \\
\hline$R_{\mathrm{FILM}}$ & ohm & $2.63 \mathrm{E}+05$ & $5.33 \mathrm{E}+06$ & $1.57 \mathrm{E}+06$ & $6.32 \mathrm{E}+09$ \\
\hline$Q_{\text {Substrate }}$ & $\begin{array}{c}\mathrm{S} \mathrm{s}^{\mathrm{a}} \\
{\left[\mathrm{Siemens} \mathrm{sec}^{\mathrm{a}}\right]}\end{array}$ & $1.63 \mathrm{E}-10$ & 5.36E-08 & $4.81 \mathrm{E}-12$ & $1.05 \mathrm{E}-08$ \\
\hline$a_{\text {Substrate }}$ & - & 1 & $1.65 \mathrm{E}-01$ & 1 & $1.86 \mathrm{E}-01$ \\
\hline Thickness & $\mathrm{nm}$ & 20 & 16 & 34 & 33 \\
\hline
\end{tabular}

Table S2. Geometry elements of the IDE used to calculate conductivity.

\begin{tabular}{ccc}
\hline IDE Features & Value & Units \\
\hline Number of Electrodes $(N)$ & 80 & - \\
Distance Between Electrodes $(d)$ & 1 & $\mu \mathrm{m}$ \\
Length of Overlap $(l)$ & 20 & $\mu \mathrm{m}$ \\
\hline
\end{tabular}

Table S3. Calculated conductivity and $C$ data.

\begin{tabular}{ccc}
\hline & Conductivity $(\mathrm{mS} / \mathrm{cm})$ & $\mathrm{C}(\mathrm{pF})$ \\
\hline Connected & $2.2( \pm 0.2)$ & $36( \pm 6)$ \\
Fingerprint & $0.5( \pm 0.1)$ & $234( \pm 14)$ \\
Partially Connected & $0.07( \pm 0.05)$ & $210( \pm 10)$ \\
Unconnected & $0.0006( \pm 0.0001)$ & $1613( \pm 113)$ \\
Homopolymer & $6( \pm 1)$ & $25( \pm 5)$ \\
\hline
\end{tabular}




\section{METHODS}

Synthesis of the random copolymer brush

$\mathrm{P}(\mathrm{S}-r-2 \mathrm{VP})-\mathrm{OH}$ with mono-hydroxide end group functionalization was synthesized by varying compositions of styrene and 2-vinylpyridine via nitroxide-mediated controlled radical polymerization (NMP). A representative polymerization procedure is described for the synthesis of copolymer as follows: A mixture of $\mathrm{OH}$-modified initiator, styrene and 2vinylpyridine was degassed through three freeze-thaw cycles. The mixture was heated at $160{ }^{\circ} \mathrm{C}$ for 72 hours under a $\mathrm{N}_{2}$ atmosphere. Conversion of both styrene and 2vinylpyridine in the random copolymerization was determined to be greater than $95 \%$ in all cases. The resulting copolymers were dissolved in THF and precipitated in hexanes. The $\mathrm{M}_{\mathrm{n}}$ of the terminal-OH copolymers were in the range of 2000-7500 g/mol, with a polydispersity index values of 1.2-1.6. The fraction of PS was determined by $1 \mathrm{H}$ NMR spectroscopy ${ }^{1}$.

\section{Thickness Change}

The thickness change of the BCP thickness was determined by spin coating a $42 \mathrm{~nm}$ thick BCP film on top of a Si wafer. The film was annealed inside of a nitrogen glove box at $250{ }^{\circ} \mathrm{C}$ for 10 mins. The resulting film was placed inside of a jar with MeI solution and functionalized for 24 hours. The resulting film was placed in a vacuum desiccator for 2 days prior to being immediately measured on the ellipsometer. The same film was then placed inside of the relative humidity chamber and conditioned at $25{ }^{\circ} \mathrm{C}$ and $95 \% \mathrm{RH}$ for 24 hour prior to being taken out and immediately measured via ellipsometer. The PS- $b$-P2VP annealed film was measured at $42 \mathrm{~nm}$, the methylated PS- $b-\mathrm{P} 2 \mathrm{VP} / \mathrm{NMP}+\mathrm{I}-$ at $0 \% \mathrm{RH}$ condition was measured to be $47.75 \mathrm{~nm}$, and the 
fully hydrated film was measured to be $49.81 \mathrm{~nm}$. The volume fraction of the hydrated film was determined by taking the equation below:

$$
\text { volfraction }=\frac{t-t_{P S}}{t}
$$

Where $t$ is the measured thickness and $t$ PS is the thickness of the PS domain, determined to be 21 $\mathrm{nm}$. The resulting hydrated thickness is determined to be approximately $58 \mathrm{vol} \%$. This volume change has also been observed through SEM in perpendicular lamellae films of the PS- $b$ $\mathrm{P} 2 \mathrm{VP} / \mathrm{NMP}+\mathrm{I}-$ in our previous work. ${ }^{2}$

\section{Solvent Vapor Annealing}

Solvent vapor annealing (SVA) in acetone was used to phase separate the PS- $b$-P2VP BCP prior to the conversion of the P2VP domain into P2VP/NMP+ I-. SVA was chosen in particular for this application because PS- $b$-P2VP can easily form thin film morphologies where the domains align perpendicular to the substrate surface as well as the free interface (e.g. interface that is exposed to the environment). The SVA chamber is shown in Figure $\mathrm{S} 1$. The $\mathrm{N}_{2}$ flows from the house $\mathrm{N}_{2}$ inlet through the lettered sections in sequence. The samples are placed in the main chamber e and closed. The sample is conditioned under $93 \mathrm{sccm}$ of dry $\mathrm{N}_{2}$ for $10 \mathrm{mins}$ prior to swelling. $31 \mathrm{sccm}$ of acetone saturated $\mathrm{N}_{2}$ gas and $62 \mathrm{sccm}$ of dry $\mathrm{N}_{2}$ are flown together into the film for $5 \mathrm{~min}$, then 62 sccm of actone vapor and $31 \mathrm{sccm}$ of dry for $5 \mathrm{~min}$, then $90 \mathrm{sccm}$ of acetone vapor and $3 \mathrm{sccm}$ of dry $\mathrm{N}_{2}$ for 2 hours. At the end of 2 hours, the system is immediately purged with 390 sccm of dry $\mathrm{N}_{2}$ to vitrify the assembled morphology. The sample is kept under $390 \mathrm{sccm}$ for 15 mins prior to removing the sample. 


\section{Quantification of structure parameters}

Raw SEM images were processed in python using the SciPy toolkit ${ }^{3}$. The original image is imported into a 2D array and the SEM metadata read to retrieve scale. The image can then be manually cropped or automatically cropped to isolate the active device area. Once cropped the image is denoised with a total variation algorithm as implemented in Scikit Image, which preserves edge information while removing most of the imaging noise ${ }^{4}$. This image is than locally thresholded to create a binary image of PS and P2VP domains. Any cluster of white (1) or black (0) pixels smaller than ten pixels is then identified and flipped to remove any remaining noise that may be present. This removal of small objects was often found not to be necessary but was kept across all images for consistency. At this point each separate foreground (white) domain is identified and. If an index of a domain exists at both the top and bottom of the active device area than it is considered a connected domain. Each pixel which matches the label of one of these connected domains is then colored blue to create the final image. This process is easily followed for the case of the connected morphology as is shown in Figure S3.

\section{Equivalent Circuit Model}

Table S1 shows all of the values extracted from the equivalent circuit model fit (inset of Figure 3a) of the impedance data. Each element represents a physical mechanism related to charge motion. $\mathrm{R}_{\mathrm{el}}$ is the combined parasitic resistance of the wiring for the potentiostat and the IDE electrode and has the unit of ohm. CPEDL represents the accumulation of charge at the electrodes. Due to the lack of a redox reaction at the interface, electrons and ions accumulate at the interface with no alternative means of transferring charge. $Q_{\mathrm{DL}}$ and aDL are fitting parameters of the CPEDL where the unit of $Q_{\mathrm{DL}}$ is Siemens $\sec ^{\mathrm{a}}$. The exponent a in the unit is the value of the fitting parameter $a_{\mathrm{DL}}$ that ranges from zero to one. When $a_{\mathrm{DL}}$ reaches one, the film represents a perfect capacitor and 
simplifies to Siemens sec, or Farads. $Q_{\text {Substrate }}$ and $a$ Substrate model the contributions of the $\mathrm{SiO}_{2}$ substrate. $R_{\text {film }}$ models the resistance of the BCE film and $Q_{\text {film }}$ and $a_{\text {film }}$ model the dielectric behavior of the BCE film.

Derivation of the inactive interfacial width from conductivity

In the discussion, the inactive interfacial width of the $\mathrm{BCE}$ is calculated by comparing the volume fraction of the P2VP/NMP+ I- domain through two methods 1.) structural information from SEM and 2.) conductivity information of the BCE and homopolymer from EIS.

The volume fraction of the conducting domain is related to the conductivity of the BCE and the homopolymer through Equation 1. In the connected film, the ion conduction pathway is direct and fully connected, hence $f=1$. When $f=1$, Equation 1 can be rearranged into the form:

$$
\phi_{C O N D}=\frac{\sigma_{B C E}}{\sigma_{H O M O}}
$$

Using the values $2.2 \mathrm{mS} / \mathrm{cm}$ and $6 \mathrm{mS} / \mathrm{cm}$ for $\sigma_{\mathrm{BCE}}$ and $\sigma_{\mathrm{COND}}$ respectively, $\phi_{\mathrm{COND}}$ is calculated to be 0.37 .

The width of the P2VP/NMP+ I- domain normal to the interface not contributing to ion transport (w) with the unit of nm was calculated by taking the difference in the P2VP/NMP+ I- fraction assessed using SEM micrographs and $\phi$ COND and multiplying the difference by the periodicity of the BCE. The equation is shown as below:

$$
w=\phi_{r} L_{0}-\phi_{C O N D} L_{0}
$$

Where $\phi_{\mathrm{r}}$ is the volume fraction of the P2VP/NMP+ I- domain determined from direct observation to be 0.58 , and $\mathrm{L}_{0}$ is the domain width of the PS-b-P2VP/NMP+ I- previously determined to be 28 
$\mathrm{nm},{ }^{2}$ and $\phi_{\mathrm{r}}$ assumes that the P2VP/NMP+ I- domain is not affected by the PS concentration. Using the values stated, the $\mathrm{w}$ value is calculated to be $3 \mathrm{~nm}$.

\section{Derivation of equation 4 - the linear scaling of conductivity and number of connected paths}

In the discussion, we successfully use structure arguments alone to predict the ion conductivity of the partially connected morphology. In this calculation, we make an assumption that the conductance linearly scales with the total number of ion-conducting domains. We can verify this approximation by first considering each independent ion conduction path as a resistor. Each connected path begins at one electrode and ends at the counter electrode, hence the ion conduction paths can be considered to be ion conduction pathways in parallel. The overall resistance of the film therefore can be calculated by taking the equation:

$$
\frac{1}{R_{\text {film }}}=\frac{1}{R_{\text {single path }, 1}}+\frac{1}{R_{\text {single path }, 2}}+\frac{1}{R_{\text {single path }, 3}}+\cdots=\sum_{n=1}^{n=n_{\text {total }}} \frac{1}{R_{\text {single path }, n}}
$$

Where the $R_{\text {film }}$ is the total resistance of the measured film and $R_{\text {singlepath,n }}$ is the resistance of each specific pathway.

We will now take the model of the connected morphology. If we assume that the resistance of each domain is the same, then the equation simplifies to:

$$
\frac{1}{R_{\text {film }}}=\frac{n}{R_{\text {single path }}}
$$

Where $n$ is the total number of connected pathways. Without taking into account the changes in ion conduction path, it is possible to consider that the inverse resistance of the partially connected path scales linearly to the number of connected pathways. The prediction of the partially connected pathway was done with conductivity values because conductance is inversely related to resistance. 
Therefore, the conductance of the film is related to the conductance of individual paths and the total number of connected paths through the modification of the above equation into:

$$
G_{\text {film }}=n G_{\text {single path }}
$$

Where $G_{\text {film }}$ is the conductance of the total film and $G_{\text {single path }}$ is the conductance of an individual conduction path. This form is modified to take into account that each path is not the same conduction due to variations in path length.

$$
G_{\text {film }}=\sum_{n=1}^{n=n_{\text {total }}} G_{\text {single path }, n}
$$

In the partially connected film, the conductance value of a single path is lower in comparison to the conductance of the single path of the connected morphology due to the due to the increased path length. Assuming conductivity scales linearly with the path length regardless of tortuosity,

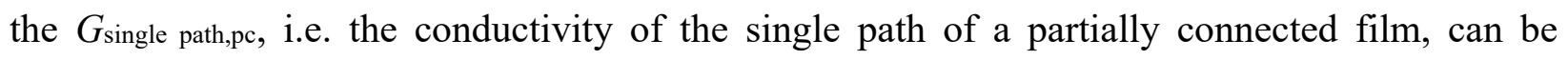
calculated by using the equation:

$$
G_{\text {single path,pc }}=\frac{l_{c}}{l_{p c}} G_{\text {single path }, c}
$$

Where $G_{\text {single _path,c }}$ is the conductance of a single path in the connected film, $l_{c}$ is the length of the path in the connected film, and $l_{\mathrm{pc}}$ is the length of the connected path of the partially connected film. Therefore the conductance of the partially connected film is related to $G_{\text {single path, }}$ by the equation:

$$
G_{p c}=\sum_{n=1}^{n=n_{\text {total }}} \frac{l_{c}}{l_{p c}} G_{\text {single path }, c}
$$


Where $G_{\mathrm{pc}}$ is the conductance of the partially connected film. To simplify the input parameters for

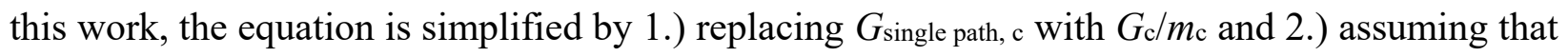
all of the connected pathways of the partially connected film have a similar length. With these assumptions, the equation becomes:

$$
G_{p c}=m_{p c} \frac{l_{c}}{l_{p c}} \frac{G_{c}}{m_{c}}
$$

Where $m_{\mathrm{c}}$ is the number of connected domains in the connected film and $m_{\mathrm{pc}}$ is the number of connected domains in the partially connected film. Rearranging the equation, one arrives at:

$$
G_{p c}=\frac{m_{p c}}{m_{c}} \frac{l_{c}}{l_{p c}} G_{c}
$$

Conductivity is calculated from conductance using the equation:

$$
\sigma=\frac{G d}{A}
$$

Where $d$ is the distance between electrodes and $A$ is the cross-sectional area of the film. Assuming these geometric parameters are identical for the connected and partially connected films, the conductivity of the partially connected film $\sigma_{\mathrm{pc}}$ can be calculated from the conductivity of the connected film $\sigma_{\mathrm{c}}$ using the equation:

$$
\sigma_{p c}=\frac{m_{p c}}{m_{c}} \frac{l_{c}}{l_{p c}} \sigma_{c}
$$

Interpretation of the C Parameter

It is important to note, when the relative permittivity of the film is calculated from the $C$ and the geometry of the $\mathrm{BCP}$ thin film, the resulting relative permittivity of the $\mathrm{BCP}$ is close to $10^{6}$. This 
is an unreasonable value in comparison to the reported relative permittivity values of PS and hydrated polymers that are 2.4 and $<88$ respectively. The discrepancy in the expected and actual values may be explained due to the presence of the thick thermal oxide below the IDE and the BCE film. It is possible that the CPEsub element is not sufficient to isolate the $C$ of the film. Still, the films may be compared to the same material and film geometry.

\section{REFERENCE}

1. Mansky, P., Liu, Y., Huang, E., Russell, T. P. \& Hawker, C. Science. 1997, 275 (5305), $1458-1460$

2. $\quad$ Arges, C. G., Kambe, Y., Suh, H. S., Ocola, L. E. \& Nealey, P. F. Chem. Mater. 2015, 28 (5), 1377-1389

3. Jones, E., Oliphant, E. \& Peterson, P. SciPy: Open Source Scientific tools for Python. (2001).

4. van der Walt, S. et al. scikit-image: image processing in Python. PeerJ 2, e453 (2014). 